
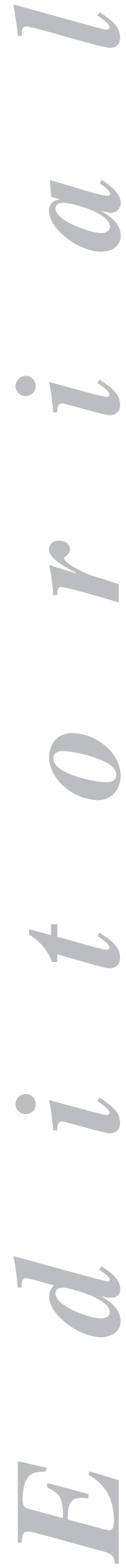

\section{New perspectives in the treatment of pediatric Crohn's disease}

Pediatric inflammatory bowel disease (PIBD) presents with a clearly increased frequency in our setting (1), and it is currently considered that up to $25 \%$ of global cases begin during childhood. PIBD has a number of specific characteristics that render its management truly challenging for pediatricians. On the one hand, its initial presentation, particularly in the case of Crohn's disease (CD), may be much more insidious than in adults. It is estimated that only $25 \%$ of pediatric $\mathrm{CD}$ cases manifest the so-called "classic triad" (abdominal pain, diarrhea, weight loss), and many cases have only few symptoms or less conspicuous manifestations (repeated fever episodes, ponderal stagnation, pubertal delay, etc.). With all this, significant diagnostic delays are not uncommon, which adds to progressive patient deterioration. On the other hand, the deleterious effects poorly controlled chronic inflammation may have on growth and development during critical life stages (mean age at disease presentation lies within pre-pubertal ages where growth potential and bone mineralization are at a maximum) may be irreversible and condition the life of these individuals permanently. To this also adds the fact that presentation forms are usually more extensive -predominance of ileocolonic forms in Crohn's disease, and of extensive colitis or pancolitis in ulcerative colitis (UC)-, more severe, with a greater tendency to progression towards complicated forms, and with a poorer response to conventional therapies (2). Classic studies have revealed that diagnosis before 40 years of age is a risk factor for poor outcomes in $\mathrm{CD}$ (poor outcome being defined as progression towards stenosing or fistulizing forms and early need for intensive therapy) (3). In order to better stratify all these differential characteristics, the Paris classification for IBD was propounded in 2011 to extend the previous Montreal classification then in force, also considering CD aspects such as presentation at early ages (A1a for cases diagnosed before 10 years of age; A1b for cases diagnosed between 10 and 17 years of age), the various degrees of small bowel involvement (more common also in pediatric forms: L4a for involvement above Treitz angle, L4b for involvement between Treitz angle and the terminal ileum), coexistence of both stenosing and fistulizing forms (B2B3 forms), and growth impairment at diagnosis (G0, no impairment; G1, impairment), as well as distinguishing extensive colitis (E3) from pancolitis (E4) and between severe presentation forms in UC ( $\mathrm{S} 0$, no severe forms; $\mathrm{S} 1$, severe forms sometime during the course of disease) (4). Lastly, a non-negligible aspect is the fact that disease manifests at ages that are key for psycho-affective maturation and personality development, which may also be impaired by a condition where morbidity may significantly condition social, school, and family relationships.

All the above implies that in pediatric IBD the often quoted "window of opportunity" available to obtain a most effective and long-lasting control is even narrower as compared to the adult. That is why in pediatric gastroenterology patient care should be optimized in order to obtain effective therapy strategies to preserve both physical and 
emotional growth and development as much as possible, and all this with an acceptable safety profile.

The advent of biologic therapies represented a radical shift in the management of IBD. The inclusion of anti-TNF $\alpha$ monoclonal antibodies in the medical armamentarium has revolutionized the treatment of diseases that still have limited therapeutic options. The high degree of efficacy shown by both infliximab and adalimumab in various scenarios, including patients poorly responsive or failing to immunosuppressive therapies, has made them -at least in theory- a highly promising option for pediatric patients. Pivotal studies assessing these drugs in a pediatric setting (the REACH study for infliximab and the IMAgINE study for adalimumab) $(5,6)$ confirmed the data sets obtained in adults, and even showed superior results, which eased their sequential approval for use against moderate-severe forms with previously failed treatments (enteral nutrition, steroids, immunosuppressants). A better understanding of these therapies and an increasingly higher experience in their use have granted them a growing prestige among pediatric gastroenterologists even for early disease, where most beneficial effects have been reported. In this regard, the theoretical advantages of these more intensive (though not aggressive) strategies for approaching pediatric Crohn's disease (the so-called top-down or accelerated step-up strategies) would include early disease stabilization, absence of complications resulting from prolonged poor management and selected therapies, such as long-term corticoid therapy, and patient growth and development facilitation.

Adalimumab, a humanized monoclonal antibody, has been traditionally used as second-line anti-TNF $\alpha$ drug for pediatric patients previously losing response to infliximab or where infliximab was contraindicated because of adverse reactions. Following authorization for pediatric use in 2013 under the same conditions as infliximab, and considering the results from the IMAgINE study, which showed a higher efficacy in infliximab-naive patients, a new era in the management of pediatric Crohn's disease starts as two anti-TNF $\alpha$ agents with proven efficacy under the same conditions are now available to be offered to our patients. In this regard, the paper "Efficacy and safety of adalimumab in the treatment of Crohn's disease in children" by Navas and colleagues, published in this issue (7), is an excellent example of adalimumab use in daily clinical practice, in different scenarios and with diverse indications. The intrinsic value of such descriptive papers on pediatric $\mathrm{CD}$ (where prospective clinical trials are challenging to carry out) is their reflecting our patients' complexity as well as the difficulties pediatric gastroenterologists have to face when managing this condition. Results from this study demonstrate the high effectiveness of adalimumab in this series of patients (up to $100 \%$ of cases at 12 weeks after therapy onset) as assessed by clinical and laboratory criteria (using activity indices), as well as by fecal calprotectin levels, an extremely sensitive marker for intestinal inflammation. One conclusion drawn by the authors is the potential influence of early drug use as compared to prior studies, and of the high proportion of anti-TNFo-naive patients included, a situation scarcely reported in the pediatric literature (8).

However, despite the positive results obtained with these therapies in pediatric Crohn's disease and their novel potentialities, we cannot lose sight of still controversial aspects regarding these drugs, including long-term safety and potential loss of response with progression, which would consequently limit therapeutic options. Anti-TNF drugs are relatively new therapies (the first case on the use of infliximab for Crohn's disease was in 1993 in a pediatric female patient) (9), hence long-term safety is unknown. The recent description of a new, nearly always fatal type of non-Hodgkin lymphoma, hepatosplenic T-cell lymphoma, in young patients (predominantly males) on concomitant treatment with anti-TNF antibodies and thiopurines (10) triggered alerts among pediatricians, 
even though its development seems to be more related to thiopurines than anti-TNF agents (cases reported with said immunosuppressants in monotherapy exist) whereas more recent reviews on the risk for other lymphomas while on anti-TNFs has found no risk increase in association with these drugs (11). As regards the potential loss of efficacy with these agents in pediatric patients, some studies find rates of up to $50 \%$ after 5 years (12). In view of these results, is an earlier use of these agents for pediatric IBD a reasonable approach to be recommended? Or should they be withheld for later rescue given the limited options available? The answer, while not entirely satisfactory if we think of Crohn's disease as the progressive, debilitating, complex condition it really is, seems obvious -pediatric patients with a torpid, poorly responsive condition deserve the best therapeutic option available in each step along the course of disease, even knowing that alternatives are limited as of today, which should further prompt research on new therapies in order to augment today's armamentarium.

Javier Martín-de-Carpi

Unit for Integral Care in Pediatric IBD. Section of Pediatric Gastroenterology, Hepatology and Nutrition. Hospital Sant Joan de Déu. Barcelona, Spain

\section{REFERENCES}

1. Martín-de-Carpi J, Rodríguez A, Ramos E, Jiménez S, Martínez-Gómez MJ, Medina E; on behalf of the SPIRIT-IBD Working Group of SEGHNP (Sociedad Española de Gastroenterología, Hepatología y Nutrición Pediátrica). Increasing incidence of pediatric inflammatory bowel disease in Spain (1996-2009): The SPIRIT registry. Inflamm Bowel Dis 2013;19:73-80.

2. Kugathasan S, Cohen S. Searching for New Clues in inflammatory bowel disease: Tell tales from pediatric IBD natural history studies. Gastroenterology 2008;135:1038-41.

3. Beaugerie L, Sokol H. Clinical, serological and genetic predictors of inflammatory bowel disease course. World J Gastroenterol 2012;18:3806-13.

4. Levine A, Griffiths A, Markowitz J, Wilson DC, Turner D, Russell RK, et al. Pediatric Modification of the Montreal Classification for inflammatory bowel disease: The Paris classification. Inflamm Bowel Dis 2011;17:1314-21.

5. Hyams J, Crandall W, Kugathasan S, Griffiths A, Olson A, Johanns J, et al. REACH Study Group. Induction and maintenance infliximab therapy for the treatment of moderate-to-severe Crohn's disease in children. Gastroenterology 2007;132:863-73.

6. Hyams JS, Griffiths A, Markowitz J, Baldassano RN, Faubion WA Jr, Colletti RB, et al. Safety and efficacy of adalimumab for moderate to severe Crohn's disease in children. Gastroenterology 2012;143:365-74.

7. Navas-López VM, Blasco-Alonso J, Girón-Fernández-Crehuet F, Serrano-Nieto MJ, Sierra-Salinas C. Efficacy and safety of adalimumab in the treatment of Crohn's disease in children. Rev Esp Enferm Dig 2013;105:579-84

8. Martín-de-Carpi J, Pociello N, Varea V. Long-term efficacy of adalimumab in paediatric Crohn's disease patients naïve to other anti-TNF therapies. J Crohns Colitis 2010;4:594-8.

9. Derkx B, Taminiau J, Radema S, Stronkhorst A, Wortel C, Tytgat G, et al. Tumour-necrosis-factor antibody treatment in Crohn's disease. Lancet 1993;342:173-4.

10. Mackey AC, Green L, Leptak C, Avigan M. Hepatosplenic T cell lymphoma associated with infliximab use in young patients treated for inflammatory bowel disease: Update. J Pediatr Gastroenterol Nutr 2009;48:386-8.

11. Targownik LE, Bernstein CN. Infectious and malignant complications of TNF inhibitor therapy in IBD. Am J Gastroenterol 2013;108:1835-42.

12. De Bie CI, Hummel Z, Kindermann A, Kokke FTM, Damen GM, Kneepkens CMF, et al. The duration of effect of infliximab maintenance treatment in paediatric Crohn's disease is limited. Aliment Pharmacol Ther 2011;33:243-50. 